\title{
Orbital angular momentum uncertainty relations of entangled two-photon states
}

\author{
Wei $\mathrm{Li}^{1,2,3, \mathrm{a}}$ and Shengmei Zhao ${ }^{1,2, \mathrm{~b}}$ \\ 1 Institute of Signal Processing and Transmission, Nanjing University of Posts and Telecommunications, Nanjing 210003, \\ Jiangsu, People's Republic of China \\ 2 Key Lab of Broadband Wireless Communication and Sensor Network, Nanjing University of Posts and \\ Telecommunications, Nanjing 210003, Jiangsu, People's Republic of China \\ 3 National Laboratory of Solid State Microstructures, Nanjing University, Nanjing 210093, People's Republic of China
}

Received 22 March 2021 / Accepted 10 August 2021 / Published online 17 August 2021

(C) The Author(s) 2021

\begin{abstract}
The inseparability of quantum correlation requires that the particles in the composite system be treated as a whole rather than treated separately, a typical example is the Einstein-Podolsky-Rosen (EPR) paradox. In this paper, we provide a theoretical study on the uncertainty relations of two kinds of bipartite observables in two-photon orbital angular momentum (OAM) entanglement, that is, the relative distance and centroid of the two photons at azimuth. We find that the uncertainty relations of the bipartite observables holds in any two-photon state, and they are separable in two-photon OAM entanglement. In addition, the entangled state behaves as a single particle in the bipartite representation. Finally, we find that the uncertainty relations of the bipartite observables can be used to manipulate the degree of the entanglement of an EPR state.
\end{abstract}

\section{Introduction}

There are many remarkable differences between a quantum particle and a classical particle, like the nonlocality, wave-particle duality, the coherent superposition of the wave-function and the collapse of the wavefunction caused by measurements. Extending these characteristics to multi-particle systems will lead to more abundant physical phenomena. One example is the two-photon entangled state which is the coherent superposition of two-photon product state. The long-distance quantum correlation introduced by nonlocality and coherent superposition, combined with wave-function collapse caused by measurement, twophoton entangled state plays an important role in the field of quantum computation and quantum information, for example quantum teleportation [1-5] and entanglement-based quantum key distribution [6-8].

One of the most important principle for quantum measurement that is different from classical measurement is the Heisenberg uncertainty relation [9-11], which states that the incompatible observables in one quantum particle cannot be determined simultaneously. While for a two-particle entangled state, the inseparability leads to a counterintuitive uncertainty behavior of a single particle. For example in the Einstein-PodolskyRosen (EPR) state $[12,13]$, the position and momentum which are incompatible observable of one particle can

\footnotetext{
${ }^{a}$ e-mail: alfred_wl@njupt.edu.cn

b e-mail: zhaosm@njupt.edu.cn (corresponding author)
}

be totally inferred by the measurement of the other. Actually, this is, in essence, a quantum behavior, which is caused by the coherence of two-particle state. This behavior has also been reformulated as either quantum steering [14-16] or observer-dependent uncertainty relation that plays a vital role in quantum witness [17-20]. As we learn from the entangled state, the description of the movement behavior of only one particle is inadequate for a quantum correlated two particle state. In an isolated two-particle system, its behavior always obey the uncertainty principle of quantum mechanics regardless of the state, like in quantum correlation imaging a high spatial resolution can be obtained by a higher dimensional momentum entanglement [21,22], the converse correlation between time and frequency domain for a spontaneous parametric down conversion twophoton state [23], and the enhancement of the dimension and degree of two-photon OAM entanglement by increasing their angular position correlation [24,25].

In this work, we perform a theoretical study on uncertainty relations of OAM entangled two-photon state. First, we construct two kinds of conjugate bipartite observables for the composite system which are linear combinations of the operators of the subsystems in angular position space and OAM space, respectively. Next, we establish the commutation relation for them and analyze their uncertainty relations with computational simulations. Finally, we studied the mutual exclusion between two-photon angular position correlation and OAM correlation for entangled two-photon 
(a)

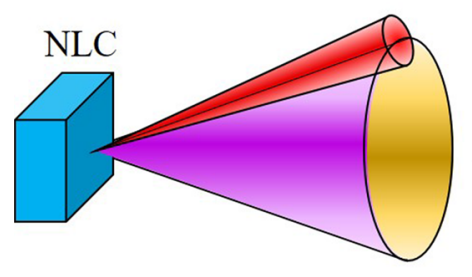

(b)

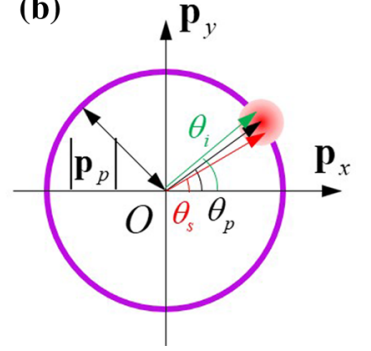

Fig. 1 Schematic illustration of SPDC two-photon correlation. a The purple cone represents a pump cone state, the red cone represents the down-converted two-photon correlation from the pump photon in the direction of the center of the red cone, NLC is a nonlinear crystal. $\mathbf{b}$ Cross section representation of SPDC of the pump cone state in momentum space

state. Modulating the entanglement of the two-photon state by using the uncertainty relations of the bipartite observables has also been discussed in detail.

\section{Uncertainty relation for two-photon correlation}

In a degenerate spontaneous parametric down conversion (SPDC) pumped by a rotationally symmetric light beam, the down converted two photons will entangle in both radial mode and azimuthal mode [26-28]. If only the azimuthal modes are concerned, a better way is to decompose the pump state into a set of cone states, which is shown as a purple cone in Fig. 1a, and the red cone represents the down-converted two-photon state in the direction of the center of the red cone. A significant advantage of this treatment is that the complex radial entanglement can be ignored. In angular position representation as shown in Fig. 1b, by considering the rotational symmetry of the pump cone state, the downconverted two-photon state can be expressed as [24]

$$
\left|\Psi\left(\theta_{s}, \theta_{i}\right)\right\rangle=\iiint d \theta_{s} d \theta_{i} d \theta_{p} \Phi\left(\theta_{p}, \theta_{s}, \theta_{i}\right)\left|\theta_{s}\right\rangle\left|\theta_{i}\right\rangle
$$

where $\theta_{p}, \theta_{s}$ and $\theta_{i}$ are the angular positions of the pump state $(p)$, the signal state $(s)$ and the idler state $(i)$, respectively; $\Phi\left(\theta_{p}, \theta_{s}, \theta_{i}\right)$, determined by the phase matching condition [29], is the angular position correlation function of the down-converted two-photon state from the pump state at $\theta_{p}$. Under paraxial approximation and assuming that the correlation scale in the transverse plane is far smaller than the radius of the pump cone state, $\Phi\left(\theta_{p}, \theta_{s}, \theta_{i}\right)$ can be approximated to $[24]$

$$
\begin{aligned}
& \Phi\left(\theta_{s}, \theta_{i}, \theta_{p}\right) \approx A \\
& \times \operatorname{sinc}\left[\frac{\left(\theta_{s}-\theta_{p}\right)^{2}+\left(\theta_{i}-\theta_{p}\right)^{2}}{\Gamma}\right] \\
& \quad \exp \left[i \frac{\left(\theta_{s}-\theta_{p}\right)^{2}+\left(\theta_{i}-\theta_{p}\right)^{2}}{\Gamma}\right],
\end{aligned}
$$

where $A$ is the normalization constant, $\Gamma=\frac{8\left|\mathbf{k}_{p}\right|}{\left|\mathbf{p}_{p}\right|^{2} L}$ is the radius of down-converted two-photon cone in angular position representation with $\left|\mathbf{k}_{p}\right|$ the wave vector of the pump beam, $L$ is the propagation distance of SPDC process within the NLC and $\left|\mathbf{p}_{p}\right|$ is the radius of the pump cone state in momentum space. Because a sinc function varies slightly in the region near around $\theta_{p}$, so Eq. (2) represents a weak correlation between the signal and idler states.

According to the Fourier relation between angular position and OAM [30]

$$
|\theta\rangle=\frac{1}{\sqrt{2 \pi}} \sum_{l=-\infty}^{\infty} \exp (-i l \theta)|l\rangle,
$$

by setting the OAM of the pump beam $l_{p}$ to 0 , we have the two-photon correlation in OAM representation

$$
\left|\Psi\left(l_{s}, l_{i}\right)\right\rangle=\frac{1}{2 \pi} \sum_{l_{s}=-\infty}^{\infty} \sum_{l_{i}=-\infty}^{\infty} \Phi\left(l_{s}, l_{i}\right) \delta_{l_{s},-l_{i}}\left|l_{s}\right\rangle\left|l_{i}\right\rangle,
$$

where $l_{s}$ and $l_{i}$ are the OAM carried by the signal state and idler state, $\Phi\left(l_{s}, l_{i}\right)$ is the two-photon OAM correlation function Fourier transformed from $\Phi\left(\theta_{s}-\theta_{p}, \theta_{i}-\theta_{p}\right)$ with respect to $\theta_{s}-\theta_{p}$ and $\theta_{i}-\theta_{p}$ [24], the delta function $\delta_{l_{s},-l_{i}}$ implies OAM conservation [29] in the down-conversion process.

As the spaces corresponding to the conjugate observables are connected by Fourier transform, now we construct the bipartite observables from the two-photon correlation function $\Phi\left(\theta_{s}-\theta_{p}, \theta_{i}-\theta_{p}\right)$ and $\Phi\left(l_{s}, l_{i}\right)$. To ease the discussion, we temporarily set $\theta_{p}$ to 0 . The exponential term connecting the two-photon correlation function in conjugate spaces can be recast into

$$
\exp \left(-i l_{s} \theta_{s}-i l_{i} \theta_{i}\right)=\exp \left(-i L_{1} \Theta_{1}-i L_{2} \Theta_{2}\right),
$$

where the observables $l_{s, i}$ and $\theta_{s, i}$ for single-photon and the observables $L_{1,2}$ and $\Theta_{1,2}$ for the joint system are defined as follows

$$
\begin{aligned}
& \hat{L}_{1}=\frac{\sqrt{2}}{2}\left(\hat{l}_{s}-\hat{l}_{i}\right), \quad \hat{L}_{2}=\frac{\sqrt{2}}{2}\left(\hat{l}_{s}+\hat{l}_{i}\right), \\
& \hat{\Theta}_{1}=\frac{\sqrt{2}}{2}\left(\hat{\theta}_{s}-\hat{\theta}_{i}\right), \hat{\Theta}_{2}=\frac{\sqrt{2}}{2}\left(\hat{\theta}_{s}+\hat{\theta}_{i}\right) .
\end{aligned}
$$

Here, the bipartite operators $L_{1}$ and $\Theta_{1}$ can be viewed as the relative distance of the two photons in azimuthal 

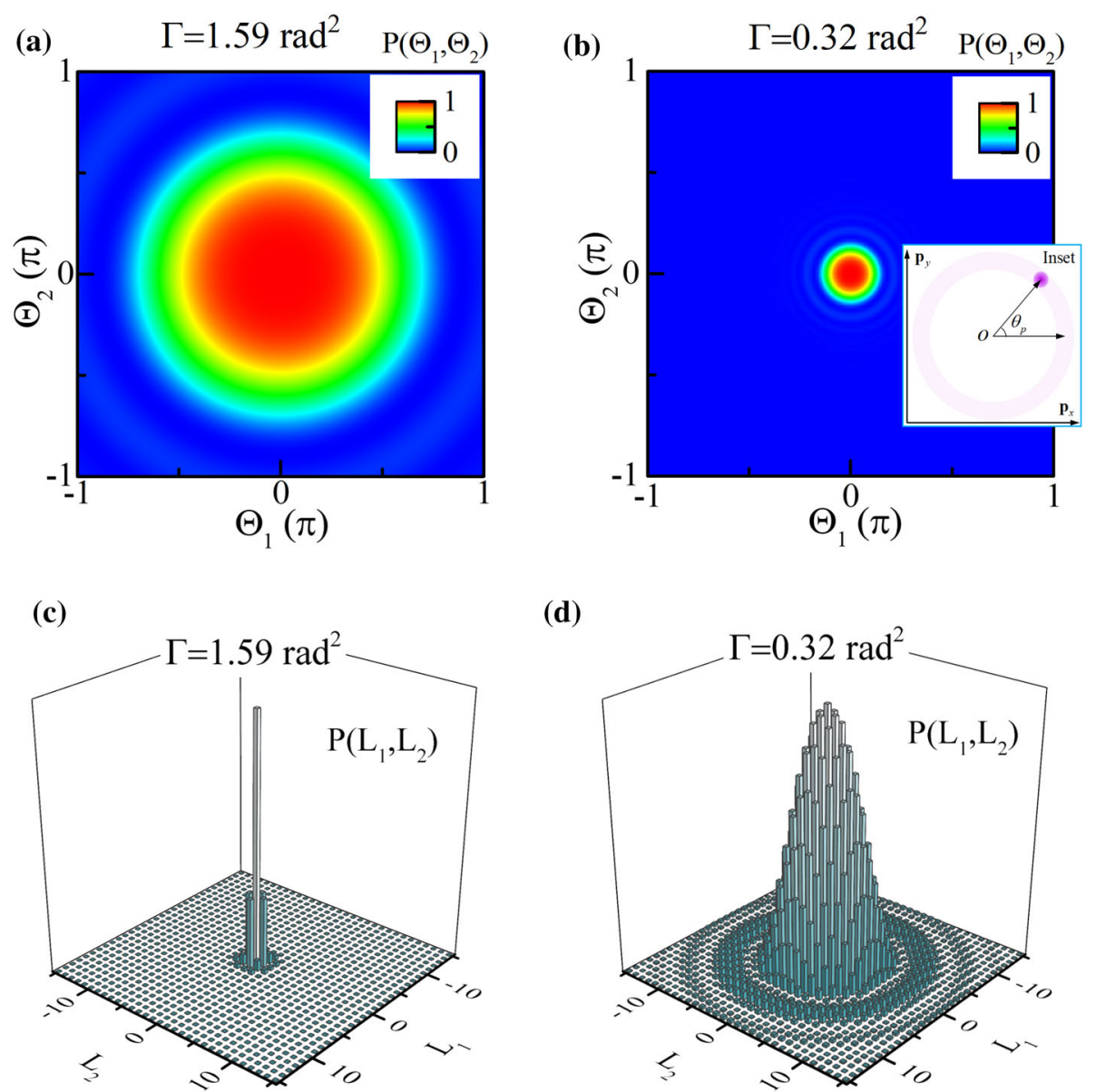

(d)

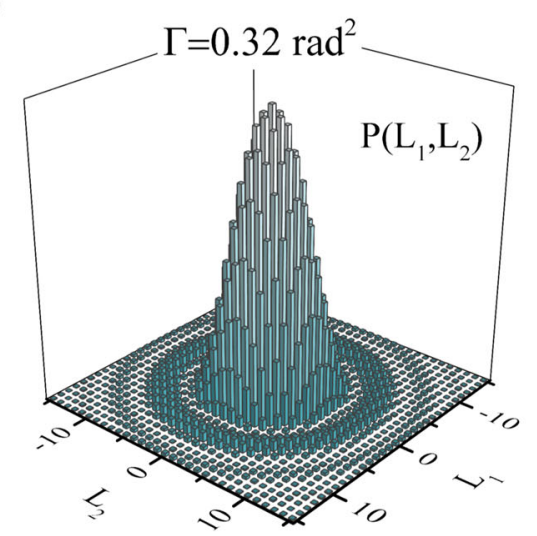

Fig. 2 Mutual exclusion between two-photon angular position correlation and OAM correlation. Joint probability distribution $\Phi\left(\Theta_{1}, \Theta_{2}\right)$ for two-photon angular position correlation for a $\Gamma=1.59 \mathrm{rad}^{2}$ and $\mathbf{b} \Gamma=0.32 \mathrm{rad}^{2}$; Joint probability distribution $\Phi\left(L_{1}, L_{2}\right)$ for $\mathbf{c}$ for two-photon OAM correlation $\Gamma=1.59 \mathrm{rad}^{2}$ and $\Gamma=0.32 \mathrm{rad}^{2}$. Inset in (b) shows that the pump light is in a point-like state at $\theta_{p}$

region, and $L_{1}$ and $\Theta_{1}$ are their centroid. Apparently, $L_{1,2}$ and $\Theta_{1,2}$ satisfy the following commutation relations

$$
\left[\hat{L}_{i}, \hat{L}_{j}\right]=\left[\hat{\Theta}_{i}, \hat{\Theta}_{j}\right]=0,\left[\hat{L}_{i}, \hat{\Theta}_{j}\right]=i \hbar \delta_{i, j}
$$

From Eqs. (5-8), we can see that $\Theta_{i}$ and $L_{i}$ are conjugate observables, the two-photon correlation function $\Phi\left(\theta_{s}, \theta_{i}\right)$ can be reformulated as $\Phi\left(\Theta_{1}, \Theta_{2}\right)$. Accordingly, the two-photon OAM correlation function $\Phi\left(L_{1}\right.$, $L_{2}$ ) can be obtained from the two-dimensional Fourier transformation of $\Phi\left(\Theta_{1}, \Theta_{2}\right)$.

According to Heisenberg uncertainty principle, the variances of $\hat{L}_{i}$ and $\hat{\Theta}_{j}$ satisfy

$$
\Delta L_{i} \Delta \Theta_{j} \geq \frac{1}{2}\left|\left\langle\left[\hat{L}_{i}, \Theta_{j}\right]\right\rangle\right|=\frac{1}{2} \hbar \delta_{i, j}
$$

where $\Delta L_{i}$ and $\Delta \Theta_{j}$ are their standard deviations. Because of the limited integration range at azimuth, the uncertainty relation is reformulated as [31-33]

$$
\Delta L_{i} \Delta \Theta_{j} \geq \frac{1}{2} \hbar \delta_{i, j}|1-2 \pi P(\Theta)|,
$$

where $P(\Theta)$ is the angular probability density at the boundary of the interval of integration. This inequality can alternatively be interpreted as mutual exclusion in determining the values of two incompatible observable.

\section{Results and discussion}

Let's first examine the general uncertainty relations for the bipartite observables in non-entangled two-photon states. In the following simulation, the down converted two-photon state is generated by a point-like pump light at $\theta_{p}$, as shown in the inset of Fig. $2 \mathrm{~b}$. The amplitude distribution of the down converted two-photon cone state is given by Eq. (2). Because of the rotational symmetry of the pump light, varying $\theta_{p}$ will only shift the two-photon state along the curve of $\theta_{s}-\theta_{i}=0$, with- 
out loss of generality, we set $\theta_{p}=0$. The joint probability distribution of two-photon states in the angularposition correlation representation and the OAM correlation representation is shown in Fig. 2. In this figure, we can see that $\Delta \Theta_{1}=\Delta \Theta_{2}$ and $\Delta L_{1}=\Delta L_{2}$, this is the common phenomenon for non-entangled twoparticle system [18]. The mutual exclusion between $\Theta_{1(2)}$ and $L_{1(2)}$ is clearly shown here. When $\Gamma$ is picked at $1.59 \mathrm{rad}^{2}$ in Fig. 2a,c, the joint probability distribution $P\left(\Theta_{1}, \Theta_{2}\right)=\left|\Phi\left(\Theta_{1}, \Theta_{2}\right)\right|^{2}$ for two-photon angular position correlation occupies a large scale, meanwhile the joint probability distribution $P\left(L_{1}, L_{2}\right)=$ $\left|\Phi\left(L_{1}, L_{2}\right)\right|^{2}$ for the two-photon OAM correlation is mainly concentrated near the zero point. While as the radius $\Gamma$ reduces to $0.32 \mathrm{rad}^{2}$ in Fig. $1 \mathrm{~b}, \mathrm{~d}$, which is 5 times smaller, the joint probability distribution $P\left(\Theta_{1}, \Theta_{2}\right)$ occupies a narrower scale, while $P\left(L_{1}, L_{2}\right)$ expands significantly.

We now extend the trivial uncertainty relationship between for the two photons in Fig. 2 to the OAM entangled state, where the pump light is in a cone state as shown in the inset of Fig. 3b. Inserting Eq. (6) in Eq. (2), we have the two-photon correlation function in the correlation representation

$$
\begin{aligned}
& \Phi\left(\Theta_{1}, \Theta_{2}-\sqrt{2} \theta_{p}\right) \approx A \\
& \quad \times \operatorname{sinc}\left[\frac{\Theta_{1}^{2}+\left(\Theta_{2}-\sqrt{2} \theta_{p}\right)^{2}}{\Gamma}\right] \exp \left[i \frac{\Theta_{1}^{2}+\left(\Theta_{2}-\sqrt{2} \theta_{p}\right)^{2}}{\Gamma}\right] .
\end{aligned}
$$

Actually, Eq. (10) is a variant of Eq. (2), where variables $\theta_{s}-\theta_{p}, \theta_{i}-\theta_{p}$ are replaced by $\Theta_{1}$ and $\Theta_{2}-\sqrt{2} \theta_{p}$ by a unitary transformation. By substituting Eq. (10) into Eq. (1), we obtain the joint probability distribution of the two-photon state in angular position correlation representation, as shown in Fig. 3a. It is the extension of the joint probability in Fig. $2 \mathrm{~b}$ along $\Theta_{1}$ axis. The joint probability distribution of the corresponding twophoton state in the OAM correlation representation is shown in Fig. 3b, which is derived from the components along the direction of $l_{p}-\sqrt{2} L_{2}=0$ in the two-photon OAM correlation spectrum $\Phi\left(L_{1}, L_{2}\right)$. In this simulation, the width $\Gamma$ is chosen as $0.32 \mathrm{rad}^{2}$, the value of $\Theta_{2}$ is evenly distributed in the range of $-\pi$ to $\pi$, while the value of $\Theta_{1}$ is concentrated near 0 with a width of $\sqrt{2} \Gamma$. The two-photon joint probability distribution in angular position space is perpendicular to that in OAM space, this is caused by the opposite correlation in conjugate spaces $[23,34]$.

Compared with Fig. 2b,d, we can see at the first glance that in Fig. 3, the values of $\Delta \Theta_{1}$ and $\Delta L_{1}$ remain unchanged, the value of $\Delta \Theta_{2}$ increases and $\Delta L_{2}$ decreases to 0 . The uncertainty relation between $L_{1}$ and $\Theta_{1}, L_{2}$ and $\Theta_{2}$ are guaranteed by Eq. (10). For $L_{2}$ and $\Theta_{2}$, since the value of $P(\Theta)$ is equal to $1 / 2 \pi$ in the range of azimuth integral of $\Theta_{2}$, we have $\Delta L_{2} \cdot \Delta \Theta_{2}=0$. Another equivalent criterion for the existence of entanglement is the violation of inequal- ity $\Delta \Theta_{1} \Delta L_{2} \geq \frac{1}{2} \hbar|1-2 \pi P(\Theta)|$, which arises from the breaking of the equalities $\Delta \Phi_{1}=\Delta \Phi_{2}$ and $\Delta L_{1}=\Delta L_{2}$ $[18,19]$. From Fig. 2, we can see that $P(\Theta)=0$ at the boundary of the azimuth integral of $\Theta_{1}$, which means that the lower bound value of Heisenberg uncertainty relation is $0.5 \hbar$. Here, $\Delta \Theta_{1} \Delta L_{2}=0$ in the simulation gives evidence of the existence of entanglement from another perspective. On the contrary, we can also get $\Delta \Theta_{2} \Delta L_{1}>\Delta \Theta_{1} \Delta L_{1} \geq \frac{1}{2} \hbar|1-2 \pi P(\Theta)|$, which is caused by quantum entanglement.

In Fig. 3b, we can see that for the pump cone state, the value of $L_{2}$ is strictly equal to 0 , which is independent of the thickness of the NLC as well as the radius of the pump cone state. In this case, the two-photon relative distance operator $L_{1}$ can be separated from the centroid operator $L_{2}$. The separability is due to the rotational symmetry of the pump cone state. In angular position correlation representation, the down-converted two-photon state generated by the pump cone state is

$$
\begin{aligned}
& \left|\Psi\left(\Theta_{1}, \Theta_{2}\right)\right\rangle \\
& \quad=\int \mathrm{d} \Theta_{1}\left|\Phi_{1}\right\rangle \int \mathrm{d} \Theta_{2}\left|\Theta_{2}\right\rangle \int_{0}^{2 \pi} \mathrm{d} \theta_{p} \Phi\left(\Theta_{1}, \Theta_{2}-\theta_{p}\right) .
\end{aligned}
$$

Because of the cyclic symmetry of the integral over $\theta_{p}$, we have the integral identity $\int_{0}^{2 \pi} \mathrm{d} \theta_{p} \Phi\left(\Theta_{1}, \Theta_{2}-\theta_{p}\right)=$ $f\left(\Theta_{1}\right)$, which is independent of $\Theta_{2}$. Then, we have the separable state for $\Theta_{1}$ and $\Theta_{2}$

$$
\left|\Psi\left(\Theta_{1}, \Theta_{2}\right)\right\rangle=\left(\int \mathrm{d} \Theta_{1}\left|\Theta_{1}\right\rangle f\left(\Theta_{1}\right)\right) \otimes \int \mathrm{d} \Theta_{2}\left|\Theta_{2}\right\rangle .
$$

Meanwhile, we have the two-photon state in OAM correlation representation

$$
\left|\Psi\left(L_{1}, L_{2}\right)\right\rangle=\left(\sum_{L_{1}=-\infty}^{\infty} g\left(L_{1}\right)\left|L_{1}\right\rangle\right) \otimes\left|L_{2}=0\right\rangle,
$$

where $g\left(L_{1}\right)$ is the Fourier transform of $f\left(\Theta_{1}\right)$. From Eqs. $(12,13)$, we can see that quantum states $\int \mathrm{d} \Theta_{2}\left|\Theta_{2}\right\rangle$ and $\left|L_{2}=0\right\rangle$ completely inherits the azimuthal modes of the pump state. So conjugate variables $\Theta_{2}$ and $L_{2}$ can be viewed as the external degrees of freedom of the two-photon state. The conjugate variables $\Theta_{1}$ and $L_{1}$, which describe the relative distances between the two photons in azimuthal region, can be viewed as the internal degrees of freedom of the two-photon state, and the entanglement is determined by the correlation functions $f\left(\Theta_{1}\right)$ and $g\left(L_{1}\right)$. If we only focus on the OAM entanglement of the two-photon state, entanglement can totally be described by conjugate variables $\Theta_{1}$ and $L_{1}$, therefore, the two-photon entanglement behaves like a single particle. The single particle behavior of two-photon correlation is totally a quantum mechanical phenomenon, and the mutual exclusion between the standard variances of $\Theta_{1}$ and $L_{1}$ is the direct result of Heisenberg's uncertainty principle. 

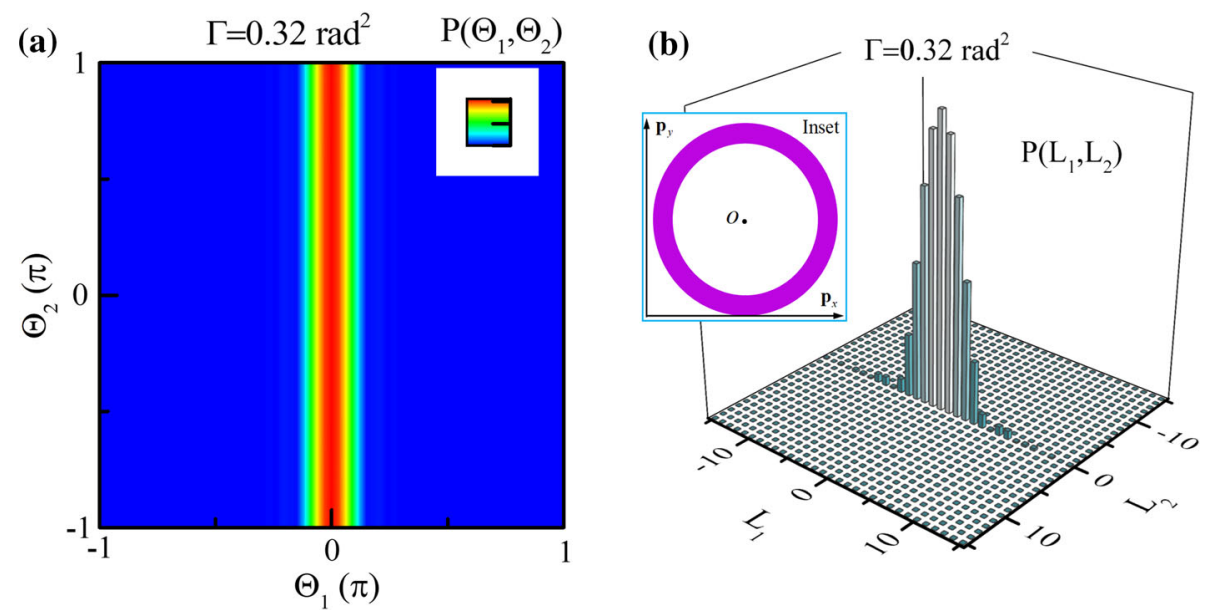

Fig. 3 Two-photon correlation spectrum in the representation of correlation operators. By the consideration of the rotational symmetry of the pump cone state, we obtain a the joint probability distribution band $P\left(\Theta_{1}, \Theta_{2}\right)$ in angular position space, $\mathbf{b}$ the corresponding joint probability distribution band $P\left(L_{1}, L_{2}\right)$ in OAM space. In this simulation, the width of two-photon angular position function $\Gamma$ is set to $0.32 \mathrm{rad}^{2}$. Inset in (b) shows that the pump light is in a cone state

Due to the single particle behavior of two-photon OAM entanglement, the mutual exclusion between $\Delta \Theta_{1}$ and $\Delta L_{1}$ is relevant to the degree of entanglement of the system. Now we study the uncertainty relation for two-photon correlation operators, and discuss its influence on the degree of entanglement of the system. Figure 4 shows the joint probability distributions of twophoton state in azimuthal region for different values of $\Gamma$. In the upper row of Fig. 4, the joint probability $P\left(\theta_{s}, \theta_{i}\right)$ rotates $\frac{\pi}{4}$ in the original azimuth representation compared with $P\left(\Theta_{1}, \Theta_{2}\right)$. The bottom row of Fig. 4 shows the corresponding two-photon OAM entangled spectrum. From this graph we can see that as $\Gamma$ reduces from $1.59 \mathrm{rad}^{2}$ to $0.16 \mathrm{rad}^{2}$, the relative distance between the two photons in angular position space is closer and, on the contrary, their relative distance in OAM space and the degree of entanglement become larger.

Next, we investigate the uncertainty relations for the two-photon relative distance operators. The standard deviation of two-photon angular position correlation is

$$
\Delta\left(\frac{\theta_{s}-\theta_{i}}{\sqrt{2}}\right)=\sqrt{\frac{\iint d \theta_{s} d \theta_{i}\left|\Phi\left(\theta_{s}, \theta_{i}\right)\right|^{2}\left(\theta_{s}-\theta_{i}\right)^{2}}{2 \iint d \theta_{s} d \theta_{i}\left|\Phi\left(\theta_{s}, \theta_{i}\right)\right|^{2}}} .
$$

Correspondingly, the standard deviation of two-photon OAM correlation is

$$
\Delta\left(\frac{l_{s}-l_{i}}{\sqrt{2}}\right)=\sqrt{\sum_{l=-\infty}^{\infty} 2 l^{2} P(l,-l)}
$$

where $P(l,-l)=|\Phi(l,-l)|^{2}$. Here, we use the Schmidt number $K$ to represent the dimension and degree of entanglement

$$
K=\frac{1}{\sum_{l} P^{2}(l,-l)},
$$

which is an estimation of the mean number of modes that participate in the entanglement. In addition, we use $H$ as the Heisenberg uncertainty function $\Delta\left(\frac{\theta_{s}-\theta_{i}}{\sqrt{2}}\right) \Delta\left(\frac{l_{s}-l_{i}}{\sqrt{2}}\right)$. When $\Gamma=1.59 \mathrm{rad}^{2}$ in Fig. 4a,b, we have the average distance between two photons in angular position space $\Delta\left(\frac{\theta_{s}-\theta_{i}}{\sqrt{2}}\right) \approx 1.1 \mathrm{rad}$, the average distance in OAM space $\Delta\left(\frac{l_{s}-l_{i}}{\sqrt{2}}\right) \approx 0.45 \hbar / \mathrm{rad}$, the Heisenberg uncertainty function $H \approx 0.5$, the Schmidt number $K \approx 1.2$. In this case, the mode distribution of two-photon OAM entanglement mainly concentrates at $l=0$. As $\Gamma$ decreases to $0.32 \mathrm{rad}^{2}$ in Fig. 4c, d, we have $\Delta\left(\frac{\theta_{s}-\theta_{i}}{\sqrt{2}}\right) \approx 0.313 \mathrm{rad}, \Delta\left(\frac{l_{s}-l_{i}}{\sqrt{2}}\right) \approx 3.82 \hbar / \mathrm{rad}$, $H \approx 1.2$, the Schmidt number $K \approx 8.3$. Now the twophoton OAM entanglement spectrum occupies a larger scale, and the average dimension reaches to 8 . When $\Gamma$ continues to decreases to $0.16 \mathrm{rad}^{2}$ in Fig. $4 \mathrm{e}$, f, we have $\Delta\left(\frac{\theta_{s}-\theta_{i}}{\sqrt{2}}\right) \approx 0.17 \mathrm{rad}, \Delta\left(\frac{l_{s}-l_{i}}{\sqrt{2}}\right) \approx 7.1 \hbar / \mathrm{rad}$, $H \approx 1.21$, the Schmidt number $K \approx 16.4$. At this time, the dimension of two-photon OAM entanglement spectrum continues to expand, and the average dimension reaches to 16.4. We can see that the Heisenberg uncertainty relations for quantum correlation for all values of $\Gamma$ are satisfied and the degree and dimension of twophoton OAM entanglement can be enlarged by increasing the strength of two-photon angular position correlation. 

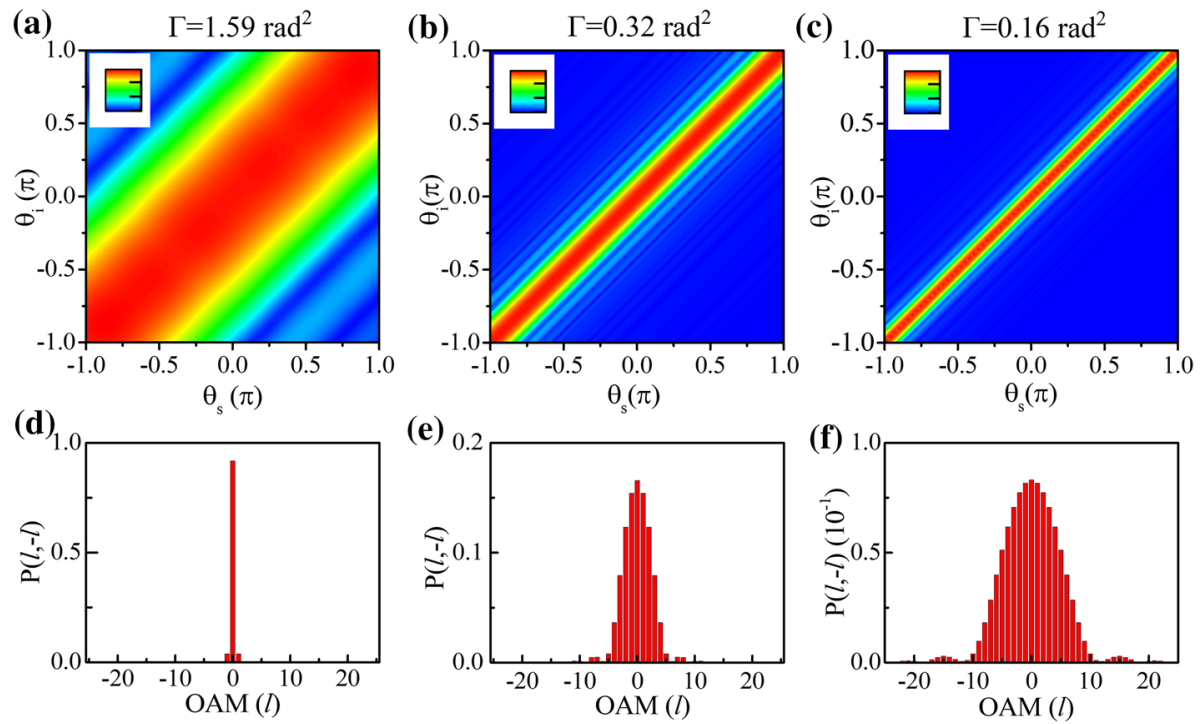

Fig. 4 Uncertainty relation for two-photon quantum correlation in azimuthal region. Joint probability distribution $P\left(\theta_{s}, \theta_{i}\right)$ in angular position space for a $\Gamma=1.59 \mathrm{rad}^{2}, \mathbf{c} \Gamma=0.32 \mathrm{rad}^{2}$, e $\Gamma=0.16 \mathrm{rad}^{2}$. The corresponding joint probability distribution $P\left(l_{s}, l_{i}\right)$ in OAM space for (b) $\Gamma=1.59 \mathrm{rad}^{2}, \mathbf{d} \Gamma=0.32 \mathrm{rad}^{2}, \mathbf{f} \Gamma=0.16 \mathrm{rad}^{2}$

\section{Conclusion}

In this paper, we have studied the uncertainty relations of bipartite observables in two-photon OAM entangled state. Two kinds of conjugate bipartite observables are defined, which can be viewed as the centroid and relative distance of the two photons at azimuth. The validity of the uncertainty relations of the bipartitee observables proved that two particles should be treated as a whole in quantum correlation. The single particle behavior of the two-photon OAM entangled state in bipartite representation has extended the concept of single particle in quantum mechanics. The uncertainty relations of bipartite observables in an EPR state may find applications in a wide range of fields, for example, the generation of a high dimensional two-photon OAM entanglement by enhancing the angular position correlation, improvement of the resolution of quantum correlation imaging by increasing the correlation size in the momentum space, and inferring the correlation range of a Cooper's pair in momentum space from the coherence length in superconductors.

Acknowledgements This work is supported by China Postdoctoral special funding project (2020T130289), the National Natural Science Foundation of China (No. 61871234).

\section{Author contributions}

Wei Li devised the theoretical scheme and provided the theoretical analysis. Wei Li and Sheng-Mei Zhao cowrote the paper.

Data Availability Statement This manuscript has no associated data or the data will not be deposited. [Authors' comment: All data can be obtained from the formulae in the article.]

Open Access This article is licensed under a Creative Commons Attribution 4.0 International License, which permits use, sharing, adaptation, distribution and reproduction in any medium or format, as long as you give appropriate credit to the original author(s) and the source, provide a link to the Creative Commons licence, and indicate if changes were made. The images or other third party material in this article are included in the article's Creative Commons licence, unless indicated otherwise in a credit line to the material. If material is not included in the article's Creative Commons licence and your intended use is not permitted by statutory regulation or exceeds the permitted use, you will need to obtain permission directly from the copyright holder. To view a copy of this licence, visit http://creativecomm ons.org/licenses/by/4.0/.

\section{References}

1. D. Bouwmeester, J.-W. Pan, K. Mattle, M. Eibl, H. Weinfurter, A. Zeilinger, Experimental quantum teleportation. Nature 390(6660), 575 (1997)

2. A. Furusawa, J.L. Sørensen, S.L. Braunstein, C.A. Fuchs, H.J. Kimble, E.S. Polzik, Unconditional quantum teleportation. Science 282(5389), 706-709 (1998)

3. D. Gottesman, I.L. Chuang, Demonstrating the viability of universal quantum computation using teleportation and single-qubit operations. Nature 402(6760), 390 (1999)

4. E. Knill, R. Laflamme, G.J. Milburn, A scheme for efficient quantum computation with linear optics. Nature 409(6816), 46 (2001)

5. C.H. Bennett, D.P. DiVincenzo, Quantum information and computation. Nature 404(6775), 247 (2000) 
6. A. Poppe, A. Fedrizzi, R. Ursin, H.R. Böhm, T. Lorünser, O. Maurhardt, M. Peev, M. Suda, C. Kurtsiefer, H. Weinfurter et al., Practical quantum key distribution with polarization entangled photons. Opt. Express 12(16), 3865-3871 (2004)

7. G. Ribordy, J. Brendel, J.-D. Gautier, N. Gisin, H. Zbinden, Long-distance entanglement-based quantum key distribution. Phys. Rev. A 63(1), 012309 (2000)

8. T. Jennewein, C. Simon, G. Weihs, H. Weinfurter, A. Zeilinger, Quantum cryptography with entangled photons. Phys. Rev. Lett. 84(20), 4729 (2000)

9. H. Percy Robertson, The uncertainty principle. Phys. Rev. 34(1), 163 (1929)

10. I. Białynicki-Birula, J. Mycielski, Uncertainty relations for information entropy in wave mechanics. Commun. Math. Phys. 44(2), 129-132 (1975)

11. D. Deutsch, Uncertainty in quantum measurements. Phys. Rev. Lett. 50(9), 631 (1983)

12. A. Einstein, B. Podolsky, N. Rosen, Can quantummechanical description of physical reality be considered complete? Phys. Rev. 47(10), 777 (1935)

13. J.C. Howell, R.S. Bennink, S.J. Bentley, R.W. Boyd, Realization of the Einstein-Podolsky-Rosen paradox using momentum-and position-entangled photons from spontaneous parametric down conversion. Phys. Rev. Lett. 92(21), 210403 (2004)

14. Q.Y. He, M.D. Reid, Einstein-Podolsky-Rosen paradox and quantum steering in pulsed optomechanics. Phys. Rev. A 88(5), 052121 (2013)

15. S. Jevtic, M. Pusey, D. Jennings, T. Rudolph, Quantum steering ellipsoids. Phys. Rev. Lett. 113(2), 020402 (2014)

16. V. Händchen, T. Eberle, S. Steinlechner, A. Samblowski, T. Franz, R.F. Werner, R. Schnabel, Observation of oneway Einstein-Podolsky-Rosen steering. Nat. Photonics 6(9), $596(2012)$

17. C.-F. Li, X. Jin-Shi, X. Xiao-Ye, K. Li, G.-C. Guo, Experimental investigation of the entanglement-assisted entropic uncertainty principle. Nat. Phys. 7(10), 752 (2011)

18. S. Mancini, V. Giovannetti, D. Vitali, P. Tombesi, Entangling macroscopic oscillators exploiting radiation pressure. Phys. Rev. Lett. 88(12), 120401 (2002)

19. L.-M. Duan, G. Giedke, J. Ignacio Cirac, P. Zoller, Inseparability criterion for continuous variable systems. Phys. Rev. Lett. 84(12), 2722 (2000)

20. O. Gühne, G. Tóth, Entanglement detection. Phys. Rep. 474(1-6), 1-75 (2009)
21. M. D'Angelo, A. Valencia, M.H. Rubin, Y. Shih, Resolution of quantum and classical ghost imaging. Phys. Rev. A 72(1), 013810 (2005)

22. J. Wen, D. Shengwang, M. Xiao, Improving spatial resolution in quantum imaging beyond the Rayleigh diffraction limit using multiphoton w entangled states. Phys. Lett. A 374(38), 3908-3911 (2010)

23. R.-B. Jin, T. Saito, R. Shimizu, Time-frequency duality of biphotons for quantum optical synthesis. Phys. Rev. Appl. 10(3), 034011 (2018)

24. W. Li, S. Zhao, Manipulating orbital angular momentum entanglement by using the Heisenberg uncertainty principle. Opt. Express 26(17), 21725-21735 (2018)

25. W. Li, S. Zhao, Generation of two-photon orbitalangular-momentum entanglement with a high degree of entanglement. Appl. Phys. Lett. 114(4), 041105 (2019)

26. C.K. Law, J.H. Eberly, Analysis and interpretation of high transverse entanglement in optical parametric down conversion. Phys. Rev. Lett. 92(12), 127903 (2004)

27. J.P. Torres, A. Alexandrescu, L. Torner, Quantum spiral bandwidth of entangled two-photon states. Phys. Rev. A 68(5), 050301 (2003)

28. S. Franke-Arnold, S.M. Barnett, M.J. Padgett, L. Allen, Two-photon entanglement of orbital angular momentum states. Phys. Rev. A 65(3), 033823 (2002)

29. F.M. Miatto, A.M. Yao, S.M. Barnett, Full characterization of the quantum spiral bandwidth of entangled biphotons. Phys. Rev. A 83(3), 033816 (2011)

30. E. Yao, S. Franke-Arnold, J. Courtial, S. Barnett, M. Padgett, Fourier relationship between angular position and optical orbital angular momentum. Opt. Express 14(20), 9071-9076 (2006)

31. S. Franke-Arnold, S.M. Barnett, E. Yao, J. Leach, J. Courtial, M. Padgett, Uncertainty principle for angular position and angular momentum. New J. Phys. 6(1), $103(2004)$

32. J.-W. Pan, Z.-B. Chen, L. Chao-Yang, H. Weinfurter, A. Zeilinger, M. Żukowski, Multiphoton entanglement and interferometry. Rev. Mod. Phys. 84(2), 777 (2012)

33. S.M. Barnett, D.T. Pegg, Quantum theory of rotation angles. Phys. Rev. A 41(7), 3427 (1990)

34. W. Li, S. Zhao, Bell's inequality tests via correlated diffraction of high-dimensional position-entangled twophoton states. Sci. Rep. 8(1), 4812 (2018) 